\title{
Learning Needs Assessment Related to Management of Diabetes: A systematic review
}

\author{
Sari Alhaik ${ }^{1}$, Jafar Alkhawaldeh ${ }^{2}$, Ahmad Aqel ${ }^{3}$, Dania Bni Hani ${ }^{4}$ \\ ${ }^{I}$ (critical care nurse, $R N, M S C /$ Applied science university, Tx.Houston ,USA) \\ ${ }^{2}$ (critical care nurse, $R N, M S C / P h D$ practitioner student, UTS University, Amman, Jordan) \\ ${ }^{3}(R N, P h D$ Lecturer, school of nursing university of Jordan, Amman, JORDAN) \\ ${ }^{4}$ (Nursing Student, the university of Jordan, AMMAN ,Jordan)
}

\begin{abstract}
Diabetes Mellitus (DM) is a chronic metabolic disorder, which is related to multiple etiologies that lead to defects of insulin secretion, insulin action or a combination of both. diabetic patients have an inadequate level of knowledge about DM will lead to poor management which negatively affects patients outcomes, quality of life, and satisfaction. Therefore the purpose of this review was to investigate the patients' knowledge about diabetes mellitus and its management in different areas of knowledge which include causes, symptoms and complications of diabetes mellitus in addition to the areas of disease management which are feet care, monitoring, meal planning, exercise and diabetic medications.
\end{abstract}

Keywords: diabetic patients' knowledge gaps,diabetic patients' knowledge, , diabetic patients' self-care, Learning needs, management methods and adherence.

\section{Introduction}

The literature review was done by searching the following searching engines: MIDLINE, CINAHL, GOOGLE SCHOLAR, PUBMED, text books and online text books for the following key words: Learning needs, Diabetes mellitus for the prevalence, causes, signs and symptoms, complications and management methods, healthy diet, physical exercise, blood glucose self-monitoring, diabetic patients' knowledge, diabetic patients' knowledge gaps, knowledge and self-efficacy, diabetic patients' self-care, medications adherence. all articles that have been reviewed were published in English .

\subsection{Learning Needs Assessment:}

Need is typically defined as deficiency or lack of something requisite, desirable, or useful. Discrepancy is the preferred term for learning needs. A discrepancy is a difference, a lack of balance between the actual and the desired. It may indicate the difference between the present and the desired performance. Learning needs assessment reveal discrepancies between the present knowledge and the skill performance and the organization's standards of desired knowledge and skill performance [16].

According to Joint Commission (2012), education that will be provided to the patients should be appropriate for the patients' conditions and their level of education, and should address and detect patients' learning needs. Learning needs assessment has a vital role in producing valuable information about the gaps into patients' knowledge which is related to the patients' current health situation, in helping the health-care provider to become familiar with the patients' educational needs, and in writing the educational plan by identifying appropriate teaching strategies regarding to patients' learning needs. Learning needs can be classified into three domains: 1) knowledge, 2) attitude, and 3) skills. The knowledge domain focuses on what the patients know about given health situation, such as listing the signs and symptoms of hypoglycemia and the appropriate behavior to regulate the blood glucose level. The attitude domain reflects the patients' values and believes, such as believing that smoking is bad; therefore, they will seek to quit. The skills domain includes acquisition of new skills and the ability to perform new technique, such as dressing changes, insulin self-injection, and blood pressure measurement [10].

\subsection{Diabetes Mellitus (DM):}

DM is an endocrine disorder characterized by elevation in blood glucose level which is related to abnormality in insulin secretion, insulin's action or a combination of both. DM is classified as type one and type two; DM Type one is defined as insulin-dependent diabetes mellitus or juvenile-onset diabetes mellitus. This type of DM results from autoimmune destruction of beta cells in the pancreas which is responsible for insulin secretion in the blood stream. DM type two is defined as non-insulin-dependent diabetes mellitus or adult-onset diabetes mellitus which is related to insufficient insulin production (absolutely or relative to the body's needs) to keep up with the excessive insulin demand related to excessive calorie intake or stress and may result from increasing in the tissue resistance which is associated with obesity that make the cells unable to use insulin 
properly and efficiently. All of these causes lead to defective in insulin action that results in hyperglycemia (increase blood glucose level) and diabetes [17].

DM can be a hereditary disease which can be inherited from one generation to another. The study of [18], reported that DM can be attributed to genetic causes and emphasize that there is a link between DM and predisposing genetic factors. The treatment of diabetes mellitus relies on the type of diabetes mellitus; DM type one requires exogenous insulin in order to control blood glucose level. Without insulin treatment the blood glucose level may increase severely and lead to life-threatening complications as diabetic ketoacidosis (DKA). The management of type two DM can be achieved by following a healthy lifestyle (e.g. healthy diet and exercise, etc....); however, this type of DM requires oral hypoglycemic agents (pills) and/or using insulin injections to meet the target of blood glucose level. [19].

\subsubsection{Diagnostic laboratory tests:}

There are several available laboratory tests to diagnose diabetes mellitus, these tests measure short-term and long-term blood glucose levels. One of these tests is urinary test which can be used to test the average of glucose level or to test the presence of ketones in blood since ketones are eliminated in the urine. Urine test is not recommended for diabetic patients because too much variation exists in the threshold for glucose when diabetes-kidney damage has occurred, does not reflect glucose level in specific point of time and is altered by some medications.

Blood tests are more recommended for diabetic patient which include short-term test such as fasting blood glucose (FBG) which is simple test can be done after the person has not eaten for 8 hours, the normal range for FBG is $70-100 \mathrm{mg} / \mathrm{dl}$. The long-term tests are used to measure blood glucose levels over an extended period of time. Glycated hemoglobin test which also known as glycosylated hemoglobin $\left(\mathrm{HbA}_{1 \mathrm{C}}\right.$ or $\left.\mathrm{A}_{1 \mathrm{C}}\right)$ is a long-term test can provide information about the average of blood glucose level over the previous three to four months. A normal $\mathrm{HbA}_{1 \mathrm{C}}$ value is less than 5.4\%. This test is recommended two or three times per year[ 20].

According to [17], the diagnostic criteria for diabetes mellitus include:

Fasting plasma glucose level at or more than $7.0 \mathrm{mmol} / \mathrm{L}(126 \mathrm{mg} / \mathrm{dL})$.

Plasma glucose at or above $11.1 \mathrm{mmol} / \mathrm{L}(200 \mathrm{mg} / \mathrm{dL})$ two hours after a $75 \mathrm{~g}$ oral glucose load as in a glucose tolerance test.

Glycated hemoglobin (hemoglobin A1C) at or above $48 \mathrm{mmol} / \mathrm{mol}(\geq 6.5$ DCCT \%).

\subsubsection{Signs and symptoms associated with diabetes mellitus:}

According to [21], the most common symptoms of hyperglycemia (increase level of blood glucose) are polydepsia (excessive thirst), polyuria (frequent urination), polyphagia (excessive hunger), weight loss, blurred vision, fatigue and weakness, drowsiness, headache, abdominal pain and evidence of dehydration such as decrease in the skin turgor, tachycardia and orthostatic hypotension (decrease in blood pressure related to sudden standing up or stretching). According to [22], the symptoms of hypoglycemia (decrease blood glucose level) that result from too much insulin in the blood may be unspecific, but diabetic patients often learn to recognize their unique symptoms. The common symptoms of hypoglycemia are sweating, palpitations, tremor, hunger, behavioral changes, difficulty thinking, frank confusion, and may result in seizure, coma, and even death.

\subsubsection{Complications of diabetes mellitus:}

The associated complications with DM can be separated into acute and chronic complications. Acute life threatening consequences of DM maybe related to increase of blood glucose level such as diabetic ketoacidosis and maybe related to decrease of blood glucose level as hypoglycemia. In addition, there are longterm DM complications such as retinopathy which can lead to blindness and loss of vision, nephropathy that can end in renal failure and hypertension (high blood pressure), peripheral neuropathy with risk for foot ulcer, amputation, and abnormalities of lipoprotein metabolism that can lead to atherosclerotic cardiovascular and heart attack, peripheral arterial and cerebrovascular diseases which can lead to stroke[21].

Hypoglycemia (low blood glucose level) is an acute complication that can result from too much insulin in the blood. With mild hypoglycemia, patients suffer from feelings of weakness and hunger so they will be able to manage the hypoglycemia by consuming any sweetened foods or drinks. However, hypoglycemia can be severe and the patients become confused and may lead to loss of consciousness. In these cases the patients need hospital admission for intramuscular injections of glucagon or intravenous (IV) infusion of dextrose solution [23]. Diabetic ketoacidosis is a hyperglycemic emergency which is mostly associated with untreated high level of blood glucose in type one DM. In the absence of insulin, glucose can't enter the cells so the cells begin to metabolize fats to produce energy. The metabolism of fats produces keton bodies (toxic by-product) which accumulate in the body that may lead to dehydration, electrolytes imbalance, coma and death. The patients in this case must be admitted to the hospital and receive intravenous (IV) insulin [23]. 
A diabetic eye disease (retinopathy) is a long-term diabetic complication that can be avoided by controlling blood glucose level and regular eye examination. Retinopathy results from damaging the small blood vessels. Retinopathy may lead to vision problems and even vision loss and blindness [23]. A diabetic kidney disease (nephropathy) is a long-term diabetic complication which is related to kidney function alteration that is caused by vascular diseases. Progression of this complication may be slowed by strict controlling of blood glucose level and blood pressure. Once nephropathy has progressed and kidney function become unable to keep up with body's demand, end-stage renal diseases ensues; for this situation, dialysis or kidney transplantation are only the options left [23].Hypertension (high blood pressure) is also associated with DM. As mentioned before, diabetic nephropathy (kidney diseases) one of the common complications associated with DM. Diabetic nephropathy is the commonest cause to develop hypertension in patients with type one diabetes. Patients with type two diabetes can develop renal disease, but hypertension commonly occurs without abnormal renal function and it is often associated with obesity. Insulin resistance which is associated with type two diabetes mellitus also can be as a cause to develop hypertension by stimulating the sympathetic nervous system and the renin-angiotensin system, and promoting sodium retention which are the main causes that predispose the patients to develop hypertension [24].

Nerve disorder (neuropathy) is another microvascular (small blood vessels) complication of diabetes mellitus. In the first stage of neuropathy, diabetic patients suffer from neurological symptoms such as numbness and tingling that develop to alter the sensation and may lead to loss of feelings completely. These signs start to occur in the toes and on the soles of the feet. Less often, in more severe cases, it also involve the fingers and hands [23].Diabetic patients are vulnerable for foot ulcer (diabetic foot) which is related to several factors such as lack of diabetes monitoring and feet examination, peripheral vascular diseases that result in poor circulation and local ischemia which predisposes the patients to develop foot ulcers by delaying wound healing. Neuropathy reduces awareness of pain and trauma caused by foot wear and foreign bodies in the shoes. Neuropathy can also dry the skin and cause it to crack and make it more vulnerable for infection development. Standard advice for diabetic patients to prevent the incidence of foot ulcers include daily self-feet examination, nail care, general hygiene, care of footwear, padded socks, examining the shoes before wearing them and avoid walking barefoot. If foot ulcers exist, the patients should use sterile technique to clean the ulcers by using sterile scalpel and forceps, avoid the chemical and alcoholic agents to clean the wounds because they may damage the healthy tissue, use local antibiotic, apply dressing and change it regularly and they have to be careful that the dressing will not impede the circulation or cause further skin trauma or pressure effects [23].Diabetic patients are at risk to have abnormalities of lipoprotein metabolism that results in hyperlipidaemia (high blood fat level) regardless to their level of cholesterol. This situation is associated with a greater risk of cardiovascular disease than in non-diabetic individuals. Insulin deficiency which is associated with diabetes mellitus alter the activity of insulin-dependent enzymes such as lipoprotein lipase which results in delayed systemic clearance of certain lipids in the blood that accumulate on the vessels wall and develop atherosclerotic disorders. This abnormality in fat metabolism is more common in the type two DM which is associated with increase insulin resistance. However, this can also be seen in type one DM patients, particularly in those with poor glycemic control. Some of the lipoprotein abnormalities associated with DM are low level of high density lipoprotein (HDL) which is a good cholesterol that is engaged in blood clearance process from bad cholesterol and decrease the risk of cardiovascular diseases, increase the level of low density lipoprotein (LDL) which is a bad cholesterol that increases the risk developing cardiovascular diseases and atherosclerotic disorders; it also increases the level of lipoprotein a (Lpa) levels, which are thought to contribute to the atherothrombotic process [23].

\subsection{Management of diabetes mellitus:}

Standard management of diabetes mellitus can be achieved by following up healthy life-style and appropriate self-care which includes healthy diet, maintaining healthy weight, physical exercise, selfmonitoring, administration of insulin and oral medications, feet care and personal hygiene in order to control blood glucose within normal level and prevent complications[25].

\subsubsection{Healthy diet:}

Following up with stringent healthy diet is a critical factor required to control blood glucose level in diabetic patients, and it is a very important part of the management of diabetes mellitus and has vital role in helping the people with diabetes mellitus to maintain optimal glycemic control, healthy body weight and prevent the incidence of diabetes complications [26]. Researchers concluded that there is no one meal plan that works for all diabetic patients; however, there are guidelines that provide some information about which meals enhance glycemic control [27].

[17] recommended some guidelines for diabetic patients such as there is no ideal percentage of calories from carbohydrate, protein and fat for all people with diabetes; therefore, nutrition pattern should be based on individualized assessment of current eating pattern, preferences, and metabolic goals. The carbohydrate intake 
of vegetables, fruits, whole grains, legumes and dairy products should be advised over intake of other carbohydrates sources especially those which contain added fat, sugars or sodium. Diabetic patients should drink adequate water, unsweetened coffee or tea, reduce fruit juice intake and the sugar sweetened beverages and limiting or minimizing alcohol intake. It will be beneficial for diabetics to eat vegetables every day, including green leafy, non-starchy vegetables; generally, non-starchy vegetables have about 5 grams of carbohydrate in a 1/2 cup cooked or 1 cup raw serving. They are full of vitamins, minerals, and fiber and have very few calories (e.g. spinach, lettuce, and broccoli, beans, cabbage, carrots, Swiss chard, tomato or kale).Eating up to three servings of small fresh fruit every day (e.g. one apple 4 ozrepresents one serving which contains 15 gram of carbohydrate, 0 gram fat, 0 gram protein and 60 calories); some of least sugar fruits are cranberry, lemon and cucumber. Some of the most sugar fruits are banana, grapes and dates. It is recommended to minimize the starchy foods including; corn, potato, white rice and pasta. Diabetic patients should choose white meat, poultry and sea foods instead of red meat, choosing whole-grain bread instead of white bread and brown rice instead of white rice. Diabetic patients should choose unsaturated fats (e.g. olive oil, corn oil, or sunflower oil) instead of saturated fats (e.g. butter, ghee or animal fat).Using the hypocaloric sweeteners has the potential to reduce overall calorie and carbohydrate intake if substituted for caloric sweeteners without compensation by intake of additional calories from other food sources.

\subsubsection{Fasting and Diabetes:}

Fasting is a duty for all healthy adults and an important spiritual aspect of many religions and requires the omission of all oral intakes throughout the day time. It starts at sunrise and ends at sun set. Diabetic patients may be at risk to develop some of diabetic complications that are associated with fasting. The main risks accompanying fasting are hyperglycemia, hypoglycemia, diabetic ketoacidosis and dehydration. So diabetic patients should take into consideration some points during fasting to prevent the incidence of these complications such as, monitor blood glucose at least two time per day, consume more low glycemic index food before the fast including fruits, vegetables and salad, increase fluid intake during non-fasting hours, break the fast in case of hypoglycemia or hyperglycemia and avoid fasting on sick days [23].

\subsubsection{Carbohydrates metabolism:}

The metabolism process of the carbohydrates includes the following steps: after the foods are consumed, the carbohydrates in the food are broken down into glucose by saliva and the gut, and then the glucose enters the bloodstream. The pancreas responds to the presence of glucose by releasing insulin from beta cells (phase 1 insulin response) which allows the glucose to enter into the cells where the glucose can be used for fuel. Also, insulin releasing enhances the storage of glucose in the muscles and the liver as glycogen. If needed, the stored glycogen can later return to the blood as glucose. The remaining glucose in the blood is converted by insulin to saturated body fat. Proteins in the meal also get broken down into glucose to some degree; however, this is a much slower process than it is with carbohydrates. After the body's initial releasing of insulin, the beta cells in the pancreas start to develop new insulin which can be released as well (phase 2 insulin response).As mentioned above, if glucose is taken from the blood to the point where blood sugar levels start to approach a low level, the Pancreas starts to secrete glucagon which works to change the stored glycogen into glucose and release it into the blood stream [28].

\subsubsection{Blood glucose self-monitoring:}

Diabetes mellitus is a chronic disease, so diabetic patients should learn self-management skills which enable diabetic patients to control their blood glucose level. Self-monitoring of blood glucose level is presented as a self-management skill and is recommended as an important element in diabetes self-management [29].Daily self-Monitoring is considered as an effective way for the management of diabetes mellitus and is associated with better glycemic control regardless to the type of diabetes and the type of therapy [30]. [20] recommended three or more times per day of blood glucose self-monitoring for most patients with type one diabetes mellitus and pregnant women who are taking insulin.

Blood glucose monitoring is the main tool to check the level of diabetes controlling. This check tells the patients about blood glucose level at any time. Keeping a $\log$ of the results has a vital role to tell the health-care provider about the body's response to the current treatment plane.

Diabetic patients should be properly trained to use the blood glucose monitoring systems. Self-blood glucose monitoring includes using an electronic device that can give direct measures of blood glucose level at the time of the test. The skills of blood glucose self-monitoring include: washing the hands, inserting the strip into the meter, using the lancing device on the side of the fingertip to get a drop of blood and wait for the results on the meter's display. The patients have to know how to interpret the results of the test and know the appropriate action regarding to the test's result. This type of monitoring is useful because it needs small blood recruitments (drop of blood), fast analysis time and can be done at any time by the patients or their family 
members [31].Self-Monitoring gives regular feedback to the patients and reveals the pattern of blood glucose changes and it is useful in decision making about adjusting insulin or other medications dosage, modifying meal planning and the period of physical activity [32].

\subsubsection{Exercise and Physical activity:}

One of the most effective options in diabetes management is doing adequate physical activity and exercise in order to control blood glucose level, improve insulin action, improve the metabolism of proteins and fat and maintain healthy body weight [33].Doing moderate intensity physical activity such as brisk walking for at least 30 minutes per day is an important component of life style modifications to reduce the risk of diabetes mellitus [34]. Ideally, [35], recommended that diabetic patients have to do at least $150 \mathrm{~min} /$ week (e.g. $30 \mathrm{~min}$ on at least 5 days each week) of moderate activity including aerobics, resistance and flexibility training.

\subsubsection{Medications adherence:}

The medications of diabetes mellitus are either oral hypoglycemic agents or insulin injections. Many researches indicate that there is a strong relationship between medications adherence and glycemic control. For instance, each $10 \%$ in oral hypoglycemic agents adherence is associated with $0.1 \%$ decrease in the level of glycated hemoglobin $\left(\mathrm{Hb} \mathrm{A}_{1 \mathrm{C}}\right)$ [36].Furthermore, Donnelly and his colleagues [37]

reported that the more patients adherence to prescribed insulin therapy can achieve better glycemic control.

There are several recommendations from American Diabetes Association (2014) regarding insulin storage and syringe safety. Insulin has to be stored at room temperature not at extreme heat or extreme cold $(<36$ or $>86^{\circ} \mathrm{F},<2$ or $>30^{\circ} \mathrm{C}$ ) because injecting cold insulin maybe painful and may cause local irritation at the site of injection. Furthermore, insulin should not be stored in the freezer, direct sunlight or in the glove compartment of the car. Patients who use insulin should check its expiration date before use; insulin losses its potency after the bottle has been used for one month. The patients also have to examine the insulin in the bottle closely to make sure that the insulin looks normal without change in its color or clarity, existence of small particles, clumping, or frosting. Conventional insulin administration involves subcutaneous injection with special syringes marked in insulin units. Insulin syringes are manufactured with 0.3-, 0.5-, 1-, and 2-ml capacities. Insulin syringe must never be shared with other patients to avoid acquiring a blood-borne viral infection (e.g., acquired immune deficiency syndrome or hepatitis).

\subsubsection{Obesity and Body Mass Index (BMI):}

Obesity is a medical condition characterized by increasing body weight and excessive body fat that can result in many health problems and disorders. Obesity is globally considered the fifth risk factor for death. [38].According to [39], BMI is considered as a strong indicator to classify the people as obese. BMI is a value defined as the body weight (in kilograms) divided by the square of the body height (in meters). Normal healthy weight with regard to BMI is from 18.5 to $25 \mathrm{~kg} / \mathrm{m}^{2}$. Less than $18.5 \mathrm{~kg} / \mathrm{m}^{2}$ represents underweight and above 25 $\mathrm{kg} / \mathrm{m}^{2}$ represents overweight. Maintaining BMI within normal range by following up healthy diet and doing physical activity is an option that leads to better control of blood glucose, blood pressure and cholesterol levels and decreases the risk of the complications incidence. For instance, maintaining BMI within normal range is very significant because being overweight is one of the important lifestyle factors that are strongly associated with increasing the risk of DM incidence [40]. Scientific confirmation suggests that people with BMI more than $35 \mathrm{~kg} / \mathrm{m}^{2}$ are 20 times more likely to have DM than people with BMI within normal range 18.5 to $24.9 \mathrm{~kg} / \mathrm{m}^{2}$ [19].

\subsection{Relevant researches about diabetic patients' knowledge:}

This section includes some relevant studies conducted in different countries including Jordan. Some of these studies aimed to assess diabetic patients' knowledge related to diabetes management and the relationships between diabetic patients' knowledge and glycemic control, medications adherence and self-efficacy. Other studies were conducted with the aim to detect the gaps into diabetic patients' knowledge.

\subsubsection{Knowledge of Diabetes Management and Control by Diabetic Patients:}

A descriptive study was conducted by [25] to assess the knowledge of diabetic patients who attended the Federal Medical Center, Umuahia and Abia State, Nigeria related to diabetes management and control methodologies. According to the findings of this study, $80.2 \%$ of the subjects knew what diabetes is, its signs, symptoms and complication. Only $14.6 \%$ knew the main cause of diabetes which is the lacking of insulin. Only $4.2 \%$ knew that following up a healthy diet plan is a self-care measure to control the progression of diabetes mellitus. Based on the study's results the researcher recommended that it is necessary to establish diabetic clinic and information center to cover the gaps in diabetic patients' knowledge. In addition, nurses, doctors, dietitians 
and other health-care providers should join hands in providing diabetic patients with the right information at every available opportunity.

\subsubsection{Diabetes Knowledge, Medications Adherence and Glycemic Control among Patients with Type Two Diabetes:}

Across-sectional study was conducted by [41] to examine and investigate the relationship between the diabetic patients' knowledge and medications adherence with glycemic control in type two diabetic patients who attended the clinic of Diabetes Outpatients; Hospital Pulau Penang in Malaysia. The results of this study show that there is significant association between the variables of this study (knowledge, HbA1C, medications adherence). The results of this study indicate that the lower HbA1C is associated with higher score for knowledge and medications adherence. Furthermore, it was found that diabetic patients' knowledge about their disease is an effective factor which can lead to better glycemic control. These findings emphasized that the healthcare providers should focus their attention on patients' knowledge towards medications adherence and other factors that can affect in glycemic control.

\subsubsection{Diabetes Knowledge among Patients with Diabetes Mellitus:}

A study was conducted by [15] at King Hussein Hospital in Jordan to assess diabetic patients' knowledge. The overall results of this study concluded that the level of knowledge among diabetic patients about their disease and its management methodologies is low and this reflects that diabetic patients are not fully aware about their disease and they do not have adequate knowledge which will limit their ability to control their blood glucose level, will decrease their involvement in the management plan and may increase the possibility of health deterioration.

\subsubsection{Self-Efficacy, Self-Management, and glycemic control in Adults with Type Two Diabetes Mellitus:}

A cross-sectional study was conducted by [42] in Jordan (Amman city) at the National Diabetes Center to evaluate the relationship between diabetes self-efficacy and diabetes self-management behaviors and glycemic control with type two DM. The study reported that diet self-efficacy and using diabetic diet as a selfmanagement method enhance glycemic control; on the other hand, using insulin to maintain blood glucose level was associated with poor glycemic control. The majority of the participants have low self-efficacy and inadequate self-management behaviors. The findings of the study reported that higher self-efficacy is associated with better self-management behaviors in diet, exercise, blood sugar monitoring, and taking medications. In addition, more than half of the subjects in this study can't control their blood glucose level. Therefore, strategies to enhance and promote self-efficacy and self-care skills for diabetic patients are very essential components of diabetes education programs and are critical interventions for the patients to be able to manage their disease and prevent complications.

\subsubsection{Level and Determinants of diabetes knowledge in patients with diabetes:}

A cross-sectional descriptive study was conducted by [43] at Zimbabwe to evaluate the level of diabetic patients' knowledge, determine the main gaps in their knowledge and identify the socio-demographic and diabetes-related determinants that affect diabetes awareness and self-care practices. According to the results of the study, the major gaps in diabetic patients' knowledge were in diet, insulin use, definition of a free food, the purpose of testing $\mathrm{HbAlc}$, cause of an insulin reaction, food that should not be used to treat low blood glucose, signs of ketoacidosis and what to do when the patients start to have an insulin reaction. In addition, the incidence of diabetes complications was significantly associated with low level of diabetes knowledge.

The researcher concluded that low diabetes knowledge could be a risk factor to develop short and longterm diabetic complications. The gaps into diabetic patients' knowledge need to be covered and diabetic patients' knowledge should be improved to prevent the occurrence of diabetes-related complications and improve patients' quality of life.

\subsubsection{Evaluation of Knowledge and Self-Care Practices in Diabetic Patients and their Role in Disease Management:}

A cross sectional study was conducted by [44]to meet diabetic patients at the time of their regular visits to evaluate their knowledge and self-care practices related to diabetes management. As a result of this study about the knowledge regarding the nature of the disease, seventy eight (66.67\%) of the patients reported correct answers regarding the hereditary nature of the disease. Most of the patients $109(93,16 \%)$ answered that diabetes can be diagnosed by blood sugar examination. There were $71(61.68 \%)$ of the patients aware of the importance of the exercise in controlling the disease and 88 [75.21] of them said that diet modification is essential to control blood glucose level. 
As evidenced from this study, patients who are more aware of the disease, having knowledge and are regularly involved in self-care practices achieve better glycemic control and better management of the disease. Regular participation into health education programs makes the patients more aware regarding the disease and encourages self-care management; this reduces the risk of health deterioration and help to achieve optimal control of the disease with minimal long term complications.

\section{Conclusion}

The literature review showed a presentation about diabetes mellitus including the definition of diabetes mellitus, the differences between type one and type two diabetes mellitus and causes, signs, symptoms and the complications which are associated with this disease. In addition, literature review showed that the management of diabetes mellitus requires following up a healthy life style and commitment with management methodologies which can control blood glucose level and prevent health deterioration. Regarding to multi studies, diabetic patient should have adequate knowledge about important aspects which lead to maintain blood glucose level within normal range. These aspects include:

Healthy diet which including what kind of foods can raise blood glucose level and contribute to increase the possibility of complications occurrence and how to manage meals planning.

Self-Monitoring of blood glucose level and its importance in controlling blood glucose level and enhancing quick response to decrease the risk of complications occurrence. Moreover, diabetic patients should know what the available tests to measure blood glucose level and which the best one of them.

The physical activity and adequate period of physical exercise which diabetic patients should perform.

The importance of medications adherence and marinating healthy body weight and its essential role in decreasing the possibility of diabetes occurrence.

The literature review showed the lack of the studies that investigate diabetic patients' knowledge about diabetes mellitus and the aspects related to management of diabetes. So this study was conducted to investigate the patients' knowledge about diabetes mellitus and its management in different areas of knowledge which include causes, symptoms and complications of diabetes mellitus in addition to the areas of disease management which are feet care, monitoring, meal planning, exercise and diabetic medications.

\section{Acknowledgements}

The authors acknowledge the support from the applied science private university for the financial support granted to this research and everyone assist in this project.

[1]. world health organization. definition,diagnosis and classification of diabetes mellitus and its complications. geneva : world health organization, 1999.

[2]. diabetes mellitus.epidemiol. s.1. : world health organization, 2006. pp. 652-660.

[3]. Global burden of diabetes,prevalence,numerical estimates, and projections. king, H, Aubert, R and Herman, W. 9, 1998, diabetes care, Vol. 21, pp. 1414-1431.

[4]. Iwueze, J. Managing your diabetes:assessment and management of patients with diabetes mellitus. s.l. : owerri:skillmark media Ltd., 2007. p. 42.

[5]. contribute data to the 6th edition of the IDF Diabetes Atlas,diabetes research and clinical practice. International Diabetes Federation. 2, 2013, Vol. 100, pp. 280-281.

[6]. National Collaborating Centre for Chronic Conditions (UK), . Type 2 diabetes, National Clinical Guideline for Management in Primary and Secondary Care (Update. London: : Royal College of Physicians (UK); NICE Clinical Guidelines, No. 66, 2008.

[7]. John, M. Handbook of Diabetes Self Management. s.1. : Uyo, Bon Books Publishers, 2007. p. 11.

[8]. "Role of health education in the management of diabetes mellitus",. Afridi, M A and Khan, M N. PAKISTAN : s.n., 2003, Journal of the College of Physicians and Surgeons, Vol. 10, pp. 558-561.

[9]. Rankin, S, Stallings, K and and London, F. Patient Education in Health and Illness: Issues, Principles and Practice, (5th Edition),. Philadelphia: J : Lippincott., 2005.

[10]. Mayer, , B. "Patient and Family Education", In Urden, L. D., Stacy, K. M., Lough, M. E., (eds.) Critical Care Nursing Diagnosis and Assessment, 7th Edition. CANADA : Elsevier Mosby., 2014.

[11]. World Health Organization,. Working to ensure quality care for persons with diabetes. Geneva : : World Health Organization., 2009.

[12]. The Direct Medical Cost of Type 2 Diabetes",. Brandle, M, et al. 8, 2003, Diabetes care, Vol. 26, pp. 2300-2304.

[13]. "Diabetes and impaired glucose tolerance in Jordan: prevalence and associated risk factors",. Ajlouni, K, Jaddou, $\mathrm{H}$ and Batieha, A. 4, jordan : s.n., 1998, Journal of internal medicine, Vol. 244, pp. 317-323.

[14]. "An increase in prevalence of diabetes mellitus in Jordan over 10 years",. Ajlouni, K, et al. 5, 2008, Journal of Diabetes and its complications, Vol. 22, pp. 317-324.

[15]. "Diabetes Knowledge among Patients with Diabetes Mellitus at King Hussein Hospital",. Al-Sarihin, , K, et al. 1, JORDAN : s.n., 2012, JRMS, Vol. 19, pp. 72-77.

[16]. Abruzzese, R S. "Nursing Stuff Development Strategies for Success"., 2nd Edition.,. s.1. : Mosby Co., 1996.

[17]. American Diabetes Association . Diagnosis and classification of diabetes mellitus. s.1. : Diabetes care 37(Supplement 1): S81-S90., 2014.

[18]. "Ethnicity, Obesity, and Risk of Type 2 Diabetes in Women a 20-year follow-up study",. Sha, I, et al. 7, 2006, Diabetes care, Vol. 29, pp. 1585-1590. 
[19]. Lough, E M, et al. " Endocrine Disorders and Therapeutic Management", Critical Care Nursing Diagnosis and Assessment, (7th Edition), . CANADA : Elsevier Mosby., 2014.

[20]. American Diabetes Association,. Standards of Medical Care in Diabetes. s.1. : Diabetes Care. 2012; 35 (suppl 1):S11., 2012.

[21]. American Diabetes Association. Diagnosis and classification of diabetes mellitus. s.1. : Diabetes care, 2010. pp. 33(Supplement 1): S62-S69.

[22]. Diagnosis and classification of diabetes mellitu. s.1. : Diabetes care 28: S37., 2005.

[23]. Wass, J, Owen, K and and Turner, H. Oxford Handbook of Endocrinology and Diabetes; Diabetes Chapter, (3rd Edition). s.1. : Oxford University Press, 2014.

[24]. Diabetes and hypertension",. Lago, R M, et al. 10, 2007, Nature clinical practice Endocrinology \& metabolism, Vol. 3, pp. 667-667.

[25]. Knowledge of diabetes management and control by diabetic patients at federal medical centre umuahia abia state, Nigeria",. Okolie, U V, et al. 9, 2009, International Journal of Medicine and Medical Sciences, Vol. 1, pp. 353-358.

[26]. "Nutrition and diabetes control: advice for non-dietitians",. Dyson, P. 8, 2002, British journal of community nursing, Vol. 7, pp. 414-419.

[27]. "Nutrition therapy recommendations for the management of adults with diabetes". Evert, , A B, et al. (Supplement 1), 2014, Diabetes care, Vol. 37, pp. S120-S143.

[28]. Mckee, T and and Mackee, J R. "Carbohydrates Metabolism" in Biochemistry: The Molecular Basis of Life, 5th Edition. ENGLAND : Oxford University Press., 2012.

[29]. "Self-monitoring of blood glucose in patients with type 2 diabetes mellitus who are not using insulin",. Malanda, U L, et al. 2012, The Cochrane Library, , pp. 1465-1858.

[30]. "Self-monitoring of blood glucose levels and glycemic control", the Northern California Kaiser Permanente Diabetes registry:. Karter, A J, et al. The American journal of medicine, pp. 111-2001.

[31]. American Diabetes Association. Diabetes medication; Insulin and Oral medications. s.1. : Diabetes Car, 2015.

[32]. Beverly, , E A. Incorporating Comorbidity, Values and Preferences into Clinical Care Guidelines Designed for Older Adults Living with Type 2 Diabetes. 2008. Vol. 31, pp. S12-S54.

[33]. "The role of exercise and nutrition in type II diabetes mellitus management",. Polikandrioti, M and and Dokoutsidou, H. Athens, Greece: : s.n., 2009, Health Science Journal, p. 1: 5.

[34]. "Intensive lifestyle changes are necessary to improve insulin sensitivity: a randomized controlled trial",. Williams, , S A, Mcauley, , K A and Mann, , J I. 2002, New Zealand: Diabetes Care,, Vol. 25, pp. :445-452.

[35]. Effects of exercise on glycemic control and body mass in type 2 diabetes mellitus",a meta-analysis of controlled clinical trials. Boulé, N G, et al. 10, 2001, Jama,, Vol. 286, pp. 1218-1227.

[36]. "Oral antidiabetic medication adherence and glycemic control in managed care",. Rozenfeld, , Y, et al. 2008, U.S.A: The American Journal of Managed Care, , pp. 14:71-5.

[37]. "Adherence to Insulin and Its Association with Glycemic Control in Patients with Type 2 Diabetes",. Donnelly, L A, Morris, A D and Evans, J M. 2007, QJM,, Vol. 100:, pp. 345-50.

[38]. International Obesity Taskforce. Obesity \& Research/ Obesity the Global Epidemic. http:. www.iaso.org/iotf/ obesity/obesity the global epidemic. [Online] 2012.

[39]. World Heath Organization, . Global Database on Body Mass Index, BMI classification. . s.1. : World Health Organization. 2006. Retrieved July 27,2012, 2012.

[40]. "The global obesity pandemic: shaped by global drivers and local environments",. Swinburn, , B A, et al. (9793): , 2011, The Lancet,, Vol. 378, pp. 804-814.

[41]. "Diabetes knowledge, medication adherence and glycemic control among patients with type 2 diabetes",. Al-Qazaz, $\mathrm{H}$ K, et al. 6, Malaysia : s.n., 2011, International journal of clinical pharmacy, Vol. 33, pp. 1028-1035.

[42]. "Self-efficacy, self-management, and glycemic control in adults with type 2 diabetes mellitus",. Al-Khawaldeh, O A, Al-Hassan, M A and Froelicher, E S. 1, JORDAN : s.n., 2012, Journal of Diabetes and its Complications, Vol. 26, pp. 10-16.

[43]. "Level and determinants of diabetes knowledge in patients with diabetes in Zimbabwe", . Mufunda, , E, et al. 2012, Pan African medical journal, , p. 13.

[44]. Evaluation of Knowledge and Self-Care Practices in Diabetic Patients and their Role in Disease Management", . Padma, K, et al. 2012, India: Natl Med J, , pp. P: 3-6.

[45]. Sekaran, , U. "Sampling chapter" in "Research Methods for Bussiness", (4th Edition), United states of America:: s.1. : Hermitage Publishing Services., 2003.

[46]. "The Construct Validity of an Instrument for Measuring Type 2 Diabetes Self-Care Knowledge in Nigeria",. Adibe, M O, Aguwa, C N and Ukwe, C V. 5, 2011, Tropical journal of pharmaceutical Research,, Vol. 10, pp. 619-629.

[47]. "Factors Associated with Poor Glycemic Control among Patients with Type 2 Diabetes",. Khattab, M, et al. 2, 2010, Journal of Diabetes and its Complications, Vol. 24, pp. 84-89.

[48]. Diabetic foot care: self reported knowledge and practice among patients attending three tertiary hospital in Nigeria",. Desalu, O O, et al. 2, 2011, Nigeria: Ghana medical journal, Vol. 45.

[49]. "Effectiveness of health education program for type 2 diabetes mellitus patients attending Zagazig university diabetes clinic". Abdo, N and Mohamed, M. (3-4), 2010, Egypt:J Egypt public Health Assoc, Vol. 85, pp. 113-130.

[50]. "Public awareness of diabetes mellitus in Singapore",. Wee, H L, Ho, H K and Li, S C. 3, 2002, medical journal, Vol. 34, pp. 128134.

[51]. Sociodemographic determinants of management behavior of diabetic patients Part II, Diabetics' knowledge of the disease and their management behavior",. Kamel, N M, et al. 5, 1999, East Mediterr Health J, Vol. 5, pp. 974-983.

[52]. "Knowledge of self-care among type 2 diabetes patients in two states of Nigeria",. Jackson, I L, et al. 3, 2014, Pharmacy practice, Vol. 12, p. 404.

[53]. An assessment of the level of knowledge about diabetes mellitus among diabetic patients in a primary healthcare setting". Moodley, L and and Rambiritch, V. 10, 2007, South African Family Practice, Vol. 49, pp. 16-16d. 